\title{
Pedicle screws with a thin hydroxyapatite coating for improving fixation at the bone-implant interface in the osteoporotic spine: experimental study in a porcine model
}

\author{
Makoto Ohe, MD, Hiroshi Moridaira, MD, PhD, Satoshi Inami, MD, PhD, Daisaku Takeuchi, MD, \\ Yutaka Nohara, MD, PhD, and Hiroshi Taneichi, MD, PhD
}

Department of Orthopaedic Surgery, Dokkyo Medical University, Mibu-machi, Shimotsuga-gun, Tochigi, Japan

\begin{abstract}
OBJECTIVE Instrumentation failure caused by the loosening of pedicle screws (PSs) in patients with osteoporosis is a serious problem after spinal surgery. The addition of a thin hydroxyapatite (HA) surface coating applied by using a sputtering process was reported recently to be a promising method for providing bone conduction around an implant without a significant risk of coating-layer breakage. In this study, the authors evaluated the biomechanical and histological features of the bone-implant interface (BII) of PSs with a thin HA coating in an in vivo porcine osteoporotic spine model.

METHODS Three types of PSs (untreated/standard [STPS], sandblasted [BLPS], and HA-coated [HAPS] PSs) were implanted into the thoracic and lumbar spine (T9-L6) of 8 mature Clawn miniature pigs ( 6 ovariectomized [osteoporosis group] and 2 sham-operated [control group] pigs). The spines were harvested from the osteoporosis group at $0,2,4,8$, 12 , or 24 weeks after PS placement and from the control group at 0 or 24 weeks. Their bone mineral density (BMD) was measured by peripheral quantitative $\mathrm{CT}$. Histological evaluation of the Blls was conducted by performing bone volume/ tissue volume and bone surface/implant surface measurements. The strength of the Bll was evaluated with extraction torque testing.
\end{abstract}

RESULTS The BMD decreased significantly in the osteoporosis group $(p<0.01)$. HAPSs exhibited the greatest mean extraction peak torque at 8 weeks, and HAPSs and BLPSs exhibited significantly greater mean torque than the STPSs at 12 weeks $(p<0.05)$. The bone surface/implant surface ratio was significantly higher for HAPSs than for STPSs after 2 weeks $(p<0.05)$, and bonding between bone and the implant surface was maintained until 24 weeks with no detachment of the coating layer. In contrast, the bone volume/tissue volume ratio was significantly higher for HAPSs than for BLPSs or STPSs only at 4 weeks.

CONCLUSIONS Using PSs with a thin HA coating applied using a sputtering process strengthens bonding at the BII, which might improve early implant fixation after spinal surgery for osteoporosis. However, the absence of increased bone mass around the screw remains a concern; prescribing osteoporosis treatment to improve bone quality might be necessary to prevent fractures around the screws.

https://thejns.org/doi/abs/10.3171/2017.10.SPINE17702

KEYWORDS osteoporosis; pedicle screws; hydroxyapatite coating; osseointegration; porcine model; degenerative

$\mathrm{W}$ E have entered an era of aging society, and the use of spinal instrumentation surgery for patients with osteoporosis is increasing rapidly. ${ }^{5}$ Pedicle screws (PSs) provide the strongest biomechanical anchor in such surgery, and transpedicular fixation is now the gold standard of spinal reconstruction for deformities, traumas, tumors, and degenerative conditions. ${ }^{8}, 12$ However, the loosening of PSs, which can cause postoperative correction loss or pseudarthrosis, has been particularly evident in patients with osteoporosis and a major concern af-

ABBREVIATIONS BII = bone-implant interface; BLPS = sandblasted pedicle screw; $\mathrm{BMD}=$ bone mineral density; $\mathrm{BS}=$ bone surface; $\mathrm{BV}=$ bone volume; $\mathrm{HA}=$ hydroxyapatite; HAPS = HA-coated pedicle screw; IS = implant surface; PS = pedicle screw; ROI = region of interest; STPS = standard pedicle screw; TB = toluidine blue; TV = tissue volume.

SUBMITTED July 2, 2017. ACCEPTED October 18, 2017

INCLUDE WHEN CITING Published online March 30, 2018; DOI: 10.3171/2017.10.SPINE17702. 
ter spinal surgery. ${ }^{10}$ Most clinical studies have found low loosening rates, in some cases less than $1 \%$, after standard posterior fixation with PSs and rods with anterior support in patients without osteoporosis. ${ }^{9}$ In contrast, in a prospective randomized study, Wu et al. ${ }^{22}$ found 48 loosened PSs in a total of 464 screws in patients with osteoporotic spines. PS fixation remains a challenge for patients with severely osteoporotic spines. Previously reported methods for improving the anchoring strength of PSs in fragile bone include using expandable titanium PSs and augmentation of fixation with polymethylmethacrylate. ${ }^{22,23}$ However, these methods have not come into general use because of their technical complexity and reported adverse consequences, such as neurovascular damage caused by pedicle fracture and cement leakage.

In procedures such as the installation of titanium dental implants and total hip arthroplasty, a hydroxyapatite (HA) surface coating on the hardware used is believed to stimulate bone ingrowth at the bone-implant interface (BII), and many basic studies and clinical applications have shown that it is a powerful means of improving implant fixation. ${ }^{17,20}$ The ideal stable state of a titanium implant within bone is that of osseointegration, in which lamellar bone formed as a result of bone remodeling around the implant is in close contact with the implant without any gaps. ${ }^{2}$ Many titanium implants are coated with HA as a means of accelerating osseointegration, even in fragile bone..$^{17,20}$ The reported drawbacks of using conventional HA surface coating on implants include breakage of the coating layer, which results in detachment of the implant from the host bone. ${ }^{14}$ As a solution for coating-layer failure, a sputtering process, in which an extremely thin (approximately 1- $\mu \mathrm{m}$ ) surface coating of HA on implants prevents BII detachment while still providing bone conduction around the implant, was developed recently. ${ }^{18,19}$ We hypothesized that applying a thin HA surface coating on PSs by using the sputtering process would also enhance implant fixation strength, even in fragile osteoporotic bones. The aim of this study was to investigate the mechanical and histological states of the BII of PSs with a thin HA coating using an experimental in vivo porcine osteoporotic spine model.

\section{Methods}

\section{Implant Materials}

Monoaxial PSs made of titanium alloy (Ti-6Al-4V; Showa Ika Co., Ltd.) and with a diameter of $4.75 \mathrm{~mm}$ and length of $30 \mathrm{~mm}$ were used. Sandblasted PSs (BLPSs) were produced by sandblasting PSs to create microscopic $(0.48-$ to $1.38-\mu \mathrm{m})$ irregularities on their titanium surface, and HA-coated PSs (HAPSs) were then produced by coating the surface of BLPSs with approximately $1 \mu \mathrm{m}$ of HA by using the sputtering process. The sputtering process we used was as reported by Ozeki et al., ${ }^{19}$ which involved the application of a voltage between the BLPSs and HA powder in a vacuum into which argon gas was introduced, causing the ionized argon gas to collide with the HA powder; the ejected HA powder formed a thin film on the BLPSs. Three types of PSs were used in our experiment: HAPSs, BLPSs, and, as controls, standard PSs (STPSs) that had not undergone any surface treatment (Fig. 1).

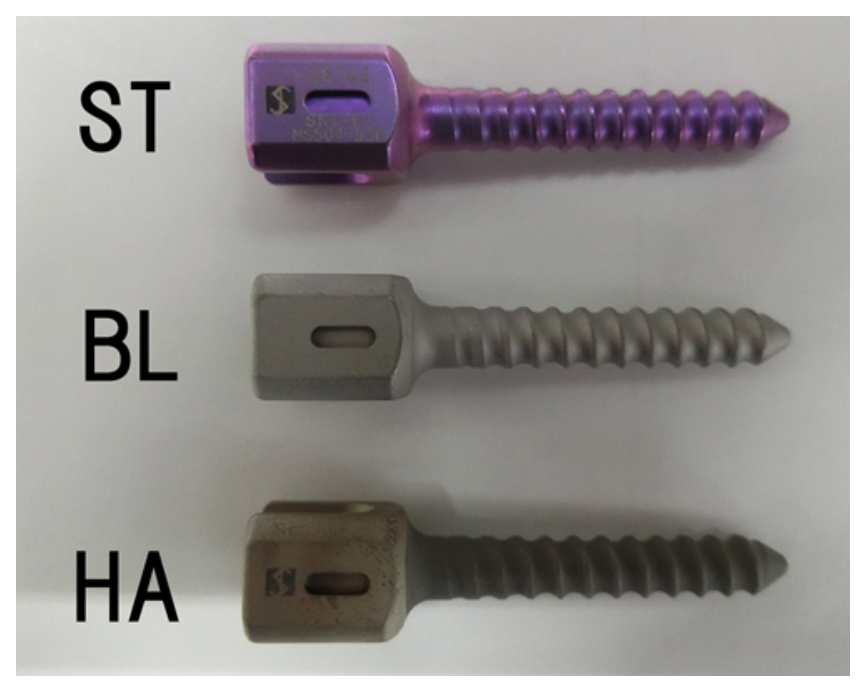

FIG. 1. Implant materials. Three types of PSs were used in this experiment: STPSs that had not undergone any surface treatment (as controls), BLPSs, and HAPSs. Figure is available in color online only.

\section{Animal Model}

This study was approved by the Dokkyo Medical University Animal Experiment Committee (experiment 0670). Eight 4-month-old female Clawn miniature pigs were used, 6 of which were used to generate an osteoporosis model following the method of Mosekilde et al. ${ }^{15}$ starting at the age of 4 months, they were fed a low-calcium diet $(0.75 \% \mathrm{Ca})$ for 6 months, underwent ovariectomy at the age of 10 months, and then were fed the same $0.75 \%$ $\mathrm{Ca}$ diet for another 6 months. Two control miniature pigs were reared, starting at the age of 4 months, on a normal diet $(0.90 \% \mathrm{Ca})$ for 6 months, underwent a laparotomic sham operation at the age of 10 months, and then were reared on the same $0.90 \% \mathrm{Ca}$ diet for another 6 months.

\section{Surgical Procedure}

Surgery for PS placement was performed when each animal was 16 months of age. After ensuring an adequate level of anesthesia, we subperiosteally exposed the thoracic and lumbar spine via a midline posterior approach. The point of insertion and the trajectory of the PSs were determined with reference to the vertebral bone specimens obtained from the same species. The 3 types of screws (STPSs, BLPSs, and HAPSs) were inserted randomly into a total of 11 vertebrae between T-10 and L-6. To avoid screw loosening on the BII, all PSs were placed independently in each vertebra and not interconnected with rods (Fig. 2). Postoperative radiographs were taken to verify the positions of the PSs (Fig. 3).

\section{Postharvest Procedure}

The osteoporosis model animals were also fed a lowcalcium diet after PS insertion, and 1 animal each was killed and their spines harvested after 0, 2, 4, 8, 12, and 24 weeks. The spines of the control animals were similarly harvested after 0 or 24 weeks. After harvest of the spine, each vertebra was split vertically along a line connecting 


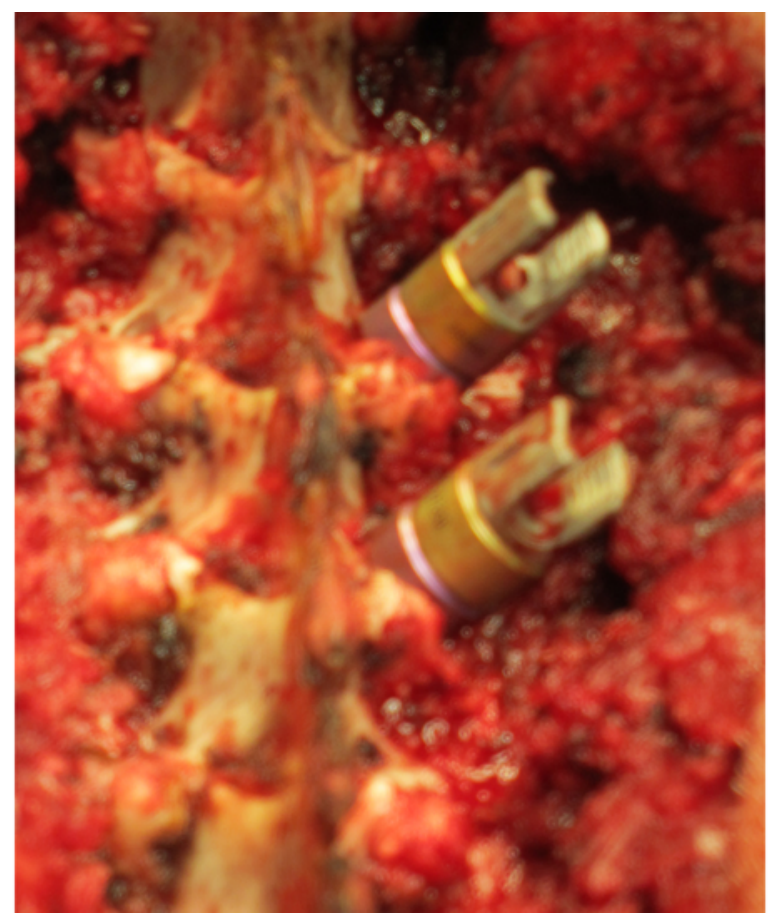

FIG. 2. Surgical procedure. All PSs were placed independently in each vertebra and not interconnected with rods. Figure is available in color online only.

the center of the spinous process and the center of the vertebral body using an oscillating saw. Vertebrae that sustained damage such as pedicle fracture or cortical breach of the PS were excluded from the study. Vertebrae T-10 and T-11 were fixed in $10 \%$ formalin for histological investigation, and a total of 9 vertebrae (T12-14 and L1-6) were immediately frozen at $-25^{\circ} \mathrm{C}$ for biomechanical testing.

\section{Measurement of Bone Mineral Density}

Five vertebrae (T5-9) were fixed in 70\% ethanol immediately after harvest. Their bone mineral density (BMD) was measured using peripheral quantitative CT (model $\mathrm{XCT}$; Stratec $\mathrm{GmbH}$ ). The analytical region was a $4 \times 6-$ $\mathrm{mm}$ area in the central portion of the vertebral body. The average bone density in this area was determined.

\section{Biomechanical Testing}

The frozen specimens (T12-L6) were defrosted to room temperature. Using metallic rods and resin, each specimen was fastened to a jig such that the screws were perpendicular. Torsional screw extraction was performed using a torque meter (model PT-1950 J; Protec) at a uniform rate $\left(360^{\circ} /\right.$ minute) to a maximum excursion of $1440^{\circ}$. The torque value and angular displacement were measured, and peak extraction torque was defined as the peak torque value in the initial linear region of the torque-angular displacement curve.

\section{Histological Analysis}

On the cross-sectional surface passing the longitudinal axis of the PS, a hard tissue-polishing sample was pre-

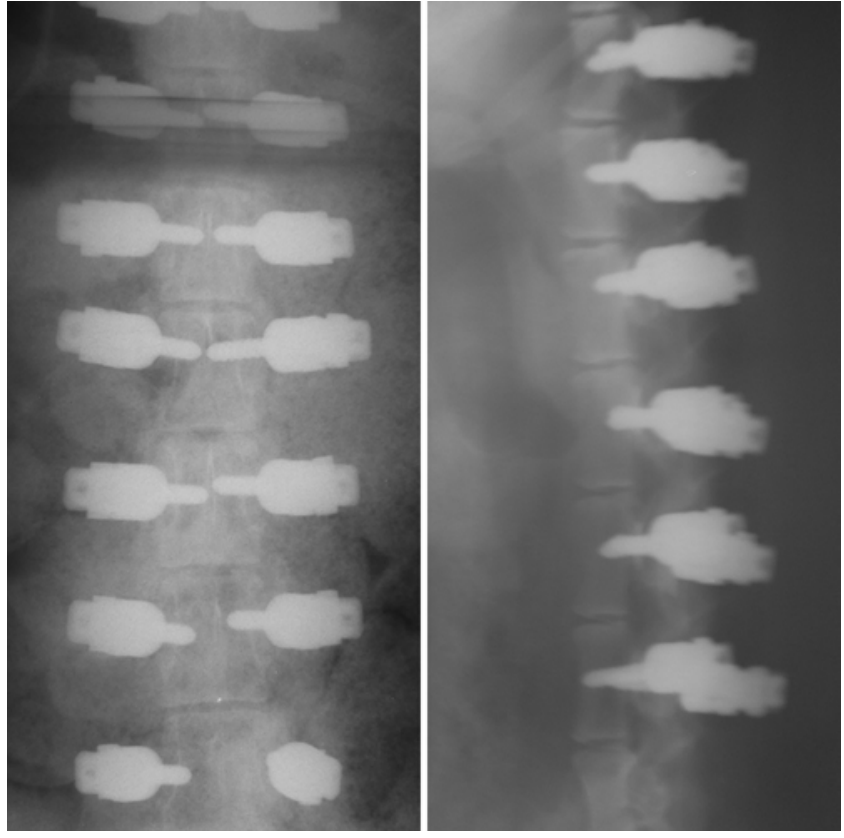

FIG. 3. Postoperative radiographs taken to verify positions of the PSs. Left: Anteroposterior view. Right: Lateral view.

pared. After cutting the specimen and embedding it with methyl methacrylate resin, we polished the observation surface with a micro grinding machine (model BS-300CL; Exakt) for mirror polishing. The slices (30-40 $\mu \mathrm{m}$ thick) were stained with $\mathrm{H} \& \mathrm{E}$ and toluidine blue (TB) stains.

Histological evaluation of the BII was carried out qualitatively and quantitatively. Qualitative evaluation was performed on the TB-stained sample in which woven bone was deeply stained. ${ }^{4}$ For the quantitative evaluation of osseointegration around the PSs, rectangular areas (1.0 $\times 1.1 \mathrm{~mm})$ that covered a valley and the adjacent threads were selected as regions of interest (ROIs). Bone volume/ tissue volume (BV/TV) and bone surface/implant surface (BS/IS) ratios were measured for each ROI (Fig. 4). ${ }^{21}$ For measurement of the BV/TV ratios, the image-processing software ImageJ (National Institutes of Health) was used, and for measurement of the BS/IS ratios, the image-analysis software WinROOF (Mitani Co.) was used.

\section{Statistical Analysis}

For statistical analysis, the Tukey-Kramer honest significant difference test and the Steel-Dwass test were used. A significant difference among the groups was defined as $p<0.05$. In contrast, nonsignificant differences among the groups was defined as $\mathrm{p}>0.20$.

\section{Results}

\section{Measurement of BMD}

All 8 experimental animals tolerated the surgery with no major complication. Table 1 lists the BMD (peripheral quantitative $\mathrm{CT}$ ) value for each animal. All members of the osteoporosis group had a BMD that was significantly lower than that of the 24-week control animal. 


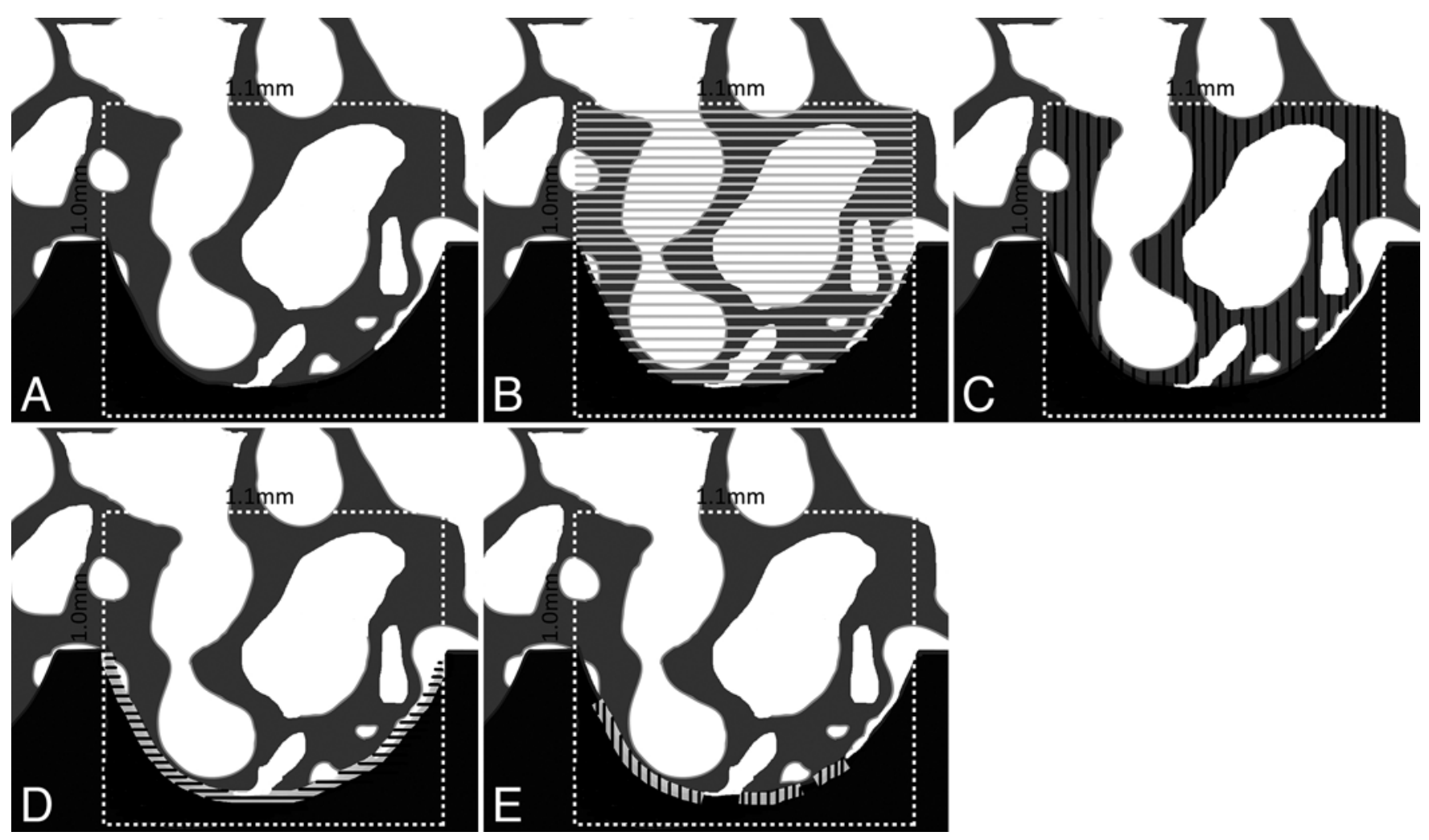

FIG. 4. A: Region of interest $(1.0 \times 1.1 \mathrm{~mm})$. B: Tissue volume: area except for the screw thread (horizontal lines). C: Bone volume: area of the bone (vertical lines). D: Implant surface: total length of the implant (band of horizontal lines). E: Bone surface: length of bone in contact with the implant (band of vertical lines).

\section{Biomechanical Testing}

A total of 134 specimens were used for biomechanical testing (Table 2). As listed in Table 3, the mean peak extraction torque was significantly higher for HAPSs than for BLPSs and STPSs in the 8-week animal. In the 12week and the 24-week control animals, the value was significantly higher for HAPSs and BLPSs than for STPSs, and in the 24-week animal, the value tended to be higher for HAPSs than for STPSs ( $p=0.076$ for HAPSs, marginally significant difference). We found no regular pattern of progression of the extraction torques over time. The

TABLE 1. BMD values for each animal

\begin{tabular}{ccc}
\hline $\begin{array}{c}\text { Time of Spine Harvest } \\
\text { (wks) }\end{array}$ & $\begin{array}{c}\text { BMD } \\
\left(\mathrm{mg} / \mathrm{cm}^{3}\right)\end{array}$ & $\begin{array}{c}\text { p Value } \\
\text { (vs 24-wk control) }\end{array}$ \\
\hline 0 & $410.6 \pm 7.2^{*}$ & $<0.0001$ \\
\hline 2 & $415.3 \pm 12.4^{*}$ & $<0.0001$ \\
\hline 4 & $451.5 \pm 12.7^{*}$ & $<0.0001$ \\
\hline 8 & $430.2 \pm 12.7^{*}$ & $<0.0001$ \\
\hline 12 & $454.6 \pm 17.5^{*}$ & $<0.0001$ \\
\hline 24 & $424.3 \pm 11.2^{*}$ & $<0.0001$ \\
\hline 0 (control) & $519.6 \pm 11.6$ & 0.2183 \\
\hline 24 (control) & $540.2 \pm 16.3$ & \\
\hline
\end{tabular}

$B M D$ values presented as the mean \pm SD.

${ }^{*} p<0.05$ compared with the 24 -week control animal. peak extraction torque in our tests was approximately 1.8 $\mathrm{Nm}$, which was the estimated plateau in 8 weeks for the HAPSs.

\section{Histological Evaluation \\ Qualitative Analysis}

In the 0-week animal, trabecular bone around the implant was disrupted and fragmented as a result of fracturing caused by screw insertion (Fig. 5A). In the 2-week animal, most of the fragmented bone had been resorbed in all samples (Fig. 5B). In the 2-week animal, some parts of the HAPS surfaces were covered with bone-lining cells comprising a single layer of osteoblasts, and some of the cells on the implant side formed woven bone (Fig. 5C), but such changes were not observed at the surface of the BLPSs and STPSs. In the 4-week animal, newly developed lamellar bone was observed at the BII of the HAPSs, and direct contact between the bone and the implant was seen (Fig. 5D), but no such increase in areas of bone substance was found in regions adjacent to the STPSs (Fig. 5E). In the 24-week animal, the formation of lamellar bone in close contact with the HAPSs was apparent (Fig. 6A). Few such areas were found with the STPSs, and numerous soft-tissue interpositions were present in the gaps between the bone and the implants (Fig. 6B). For BLPSs, although some lamellar bone was formed, it was far less than that for the HAPSs (Fig. 6C). At locations farther away from the implants, fatty marrow conversion associated with os- 
TABLE 2. Biomechanical testing specimens stratified by week when the spine was harvested

\begin{tabular}{ccccccccccc}
\hline \multirow{2}{*}{$\begin{array}{c}\text { Specimen } \\
\text { Type }\end{array}$} & 0 wk & 2 wk & 4 wk & 8 wk & 12 wk & 24 wk & 0-wk Control & 24-wk Control & Total \\
\hline HAPS & 7 & 5 & 5 & 5 & 5 & 7 & 5 & 5 & 44 \\
\hline BLPS & 5 & 5 & 5 & 6 & 6 & 7 & 5 & 5 & 44 \\
\hline STPS & 5 & 5 & 6 & 6 & 7 & 6 & 6 & 5 & 46 \\
\hline Total & 17 & 15 & 16 & 17 & 18 & 20 & 16 & 15 & 134 \\
\hline
\end{tabular}

teoporosis was present in all of the samples, and no clear difference among the 3 types of screws was seen.

\section{Quantitative Analysis}

Quantitative evaluation was carried out for all samples except for the 0 -week and 0 -week control samples, in which fragmented bone around the implants caused by PS insertion was still present. The BS/IS ratio was significantly higher for HAPSs than for STPSs in the 2-week, 4-week, and 12-week animals. In the 8-week, 24-week, and 24-week control animals, the values were significantly higher for HAPSs and BLPSs than for STPSs. In all of the samples, the value was highest for the HAPSs, and in the 24-week and 24-week control animals, it was also significantly higher for HAPSs than for BLPSs (Table 4). The BV/TV ratio was significantly higher for HAPSs than for STPSs in the 4-week animal only; we found no significant differences among the BV/TV ratios for the 3 types of screws in any of the other samples (Table 5).

\section{Discussion}

Coating ISs with HA, which possesses bone conductivity, is a useful method for achieving mechanical stability at the BII; it requires no special techniques and is comparatively safe. ${ }^{17,20}$ Several studies have addressed the use of HAPSs as a means of enhancing the fixation of PSs in fragile bone. Aldini et al. ${ }^{1}$ carried out a 16-week experiment using an osteoporotic sheep model, and Hasegawa et al. ${ }^{11}$ carried out a 10 -day experiment using a canine osteo- porosis model; both studies found that HAPSs provided an advantage over uncoated PSs. Our study is the first to have investigated the value of HAPSs at multiple time points and over a long period (from week 0 to week 24) in an osteoporotic spine model.

The plasma spray technique is currently the most widely used method of HA surface coating. However, this technique produces a thick layer of 20-100 $\mu \mathrm{m}$ that shows internal defects and degradation immediately after coating of the HA, ${ }^{13}$ and reported clinical issues include breakage of the coating layer, bone-implant detachment, and inflammatory reactions. ${ }^{14}$ Potential alternatives to the plasma spray method, including flame spraying, thermal decomposition, and sputtering, have been studied also. Ozeki et al. ${ }^{18,19}$ reported that a sputtering process can create a thin and precise HA surface coating only $1 \mu \mathrm{m}$ thick and that the use of sputtering not only prevents breakage of the coating layer but also increases the rate at which osseointegration is achieved. Ours is the first study to have investigated the value of PSs with a thin HA coating applied by using the sputtering process and implanted in osteoporotic spines.

The porcine osteoporosis model used in this study included animals with a significantly reduced BMD (76\%$84 \%$ of the values in the control animals), which suggests that it was an adequate osteoporosis model to use for evaluating implant fixation in fragile osteoporotic bone. In the extraction torque tests in this osteoporosis model, the HAPSs tended to have a higher value than the STPSs after 4 weeks; after 8 and 12 weeks, the value was signifi-

TABLE 3. Peak extraction torques of the 3 types of PSs used

\begin{tabular}{|c|c|c|c|c|c|c|}
\hline \multirow[b]{2}{*}{ Week of Spine Harvest } & \multicolumn{3}{|c|}{ Peak Extraction Torque (Nm) } & \multicolumn{3}{|c|}{$p$ Value } \\
\hline & HAPSs & BLPSs & STPSs & HAPSs vs BLPSs & HAPSs vs STPSs & BLPSs vs STPSs \\
\hline 0 wks & $0.70 \pm 0.45$ & $0.63 \pm 0.52$ & $0.68 \pm 0.38$ & 0.999 & 0.999 & 0.999 \\
\hline 2 wks & $0.51 \pm 0.55$ & $0.52 \pm 0.58$ & $0.33 \pm 0.21$ & 0.999 & 0.999 & 0.999 \\
\hline 4 wks & $1.36 \pm 0.27$ & $0.69 \pm 0.06$ & $0.35 \pm 0.39$ & 0.188 & 0.129 & 0.331 \\
\hline 8 wks & $1.88 \pm 0.24^{*} \dagger$ & $0.73 \pm 0.26$ & $0.50 \pm 0.12$ & $0.022 \ddagger$ & $0.022 \ddagger$ & 0.112 \\
\hline 12 wks & $1.32 \pm 0.21^{*}$ & $0.83 \pm 0.30^{*}$ & $0.26 \pm 0.18$ & 0.246 & $0.029 \ddagger$ & $0.033 \ddagger$ \\
\hline 24 wks & $1.74 \pm 0.73$ & $1.66 \pm 1.06$ & $0.87 \pm 0.76$ & 0.932 & 0.076 & 0.174 \\
\hline 0 wks (control) & $0.81 \pm 0.74$ & $0.90 \pm 0.63$ & $0.50 \pm 0.52$ & 0.960 & 0.806 & 0.461 \\
\hline 24 wks (control) & $1.74 \pm 073^{*}$ & $1.76 \pm 1.01^{*}$ & $0.61 \pm 0.37$ & 0.991 & $0.022 \ddagger$ & $0.048 \ddagger$ \\
\hline
\end{tabular}

Torque values presented as the mean \pm SD.

${ }^{*} p<0.05$ compared with STPSs.

$\dagger p<0.05$ compared with BLPSs.

$\ddagger$ Significant difference. 

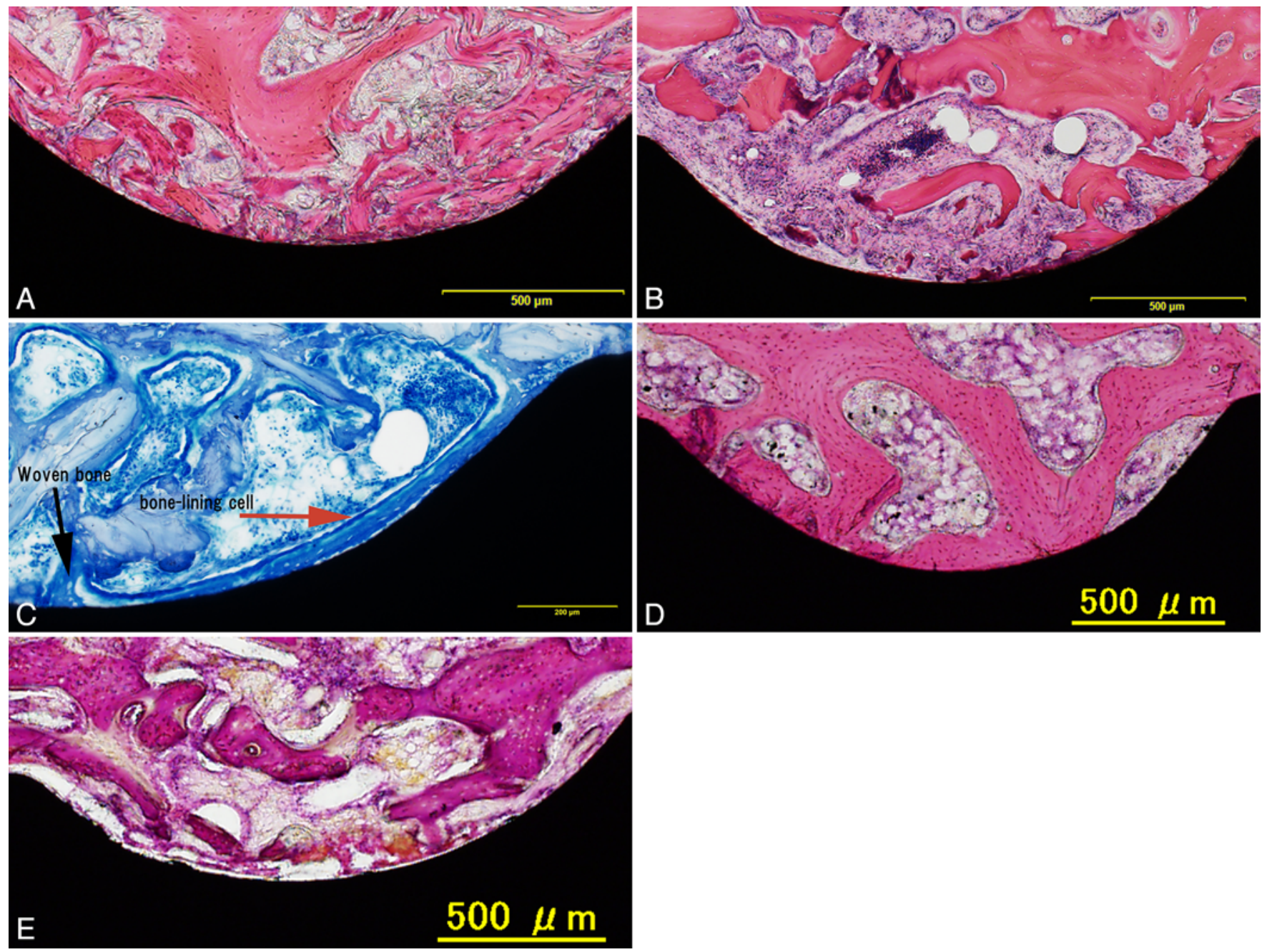

FIG. 5. Qualitative analysis of the BII. A: HAPSs in the 0-week animal (H \& E staining). Trabecular bone around the implant was disrupted and fragmented as a result of fracturing caused by screw insertion. B: HAPSs in the 2-week animal (H \& E staining). Most of the fragmented bone has been resorbed. C: HAPSs in the 2-week animal (TB staining). The IS is covered with bone-lining cells, and some of the cells have formed woven bone. D: HAPSs in the 4-week animal (H \& E staining). Newly developed lamellar bone at the BII, with direct contact between the bone and the implant. E: STPSs in the 4-week animal (H \& E staining). No increase in areas of bone substance is evident in regions adjacent to the implant. Figure is available in color online only.

cantly higher, and this tendency was maintained until 24 weeks. This result indicates that, even in fragile bone, PSs with a thin HA coating showed enhanced integration at the BII after a waiting period of approximately 8 weeks, and this integration at the BII was maintained until 24 weeks. In contrast, the extraction torque tests revealed no regular pattern of progression of the extraction torque values over time. The peak extraction torque in our tests was approximately $1.8 \mathrm{Nm}$, which might be because the BII was surrounded by cancellous bone. If osteointegration of a PS makes the BII stronger than the surrounding cancellous bone over time, the extraction torque reaches a plateau as a result of the occurrence of microfractures in the surrounding cancellous bone.

Histologically, the BS/IS ratio, which gives an indication of close contact at the BII, was significantly higher for HAPSs than for STPSs after 2 weeks. Bonding between the bone and the IS was maintained until 24 weeks, and no detachment of the coating layer or surrounding inflammation was seen (Figs. 5D and 6A). The results of our histological analysis support those from previous studies of the application of a thin HA coating to implants by using the sputtering process, ${ }^{18,19}$ and they confirmed the results of the extraction torque tests. Also, because our study was performed over multiple time points, we found that the osteoconductivity of the HA coating (seen in our histological examinations) appeared some weeks earlier than the mechanical superiority of the HAPSs was determined in the extraction torque tests.

The BLPSs had higher peak extraction torque values than did the STPSs after 12 and 24 weeks, which indicates that bone tissue invades the surface irregularities created by sandblasting and thereby increases the surface area of the BII and mechanically strengthens integration at this site, as found in previous studies. In our histological evaluation, the BLPSs had a higher BS/IS ratio than 

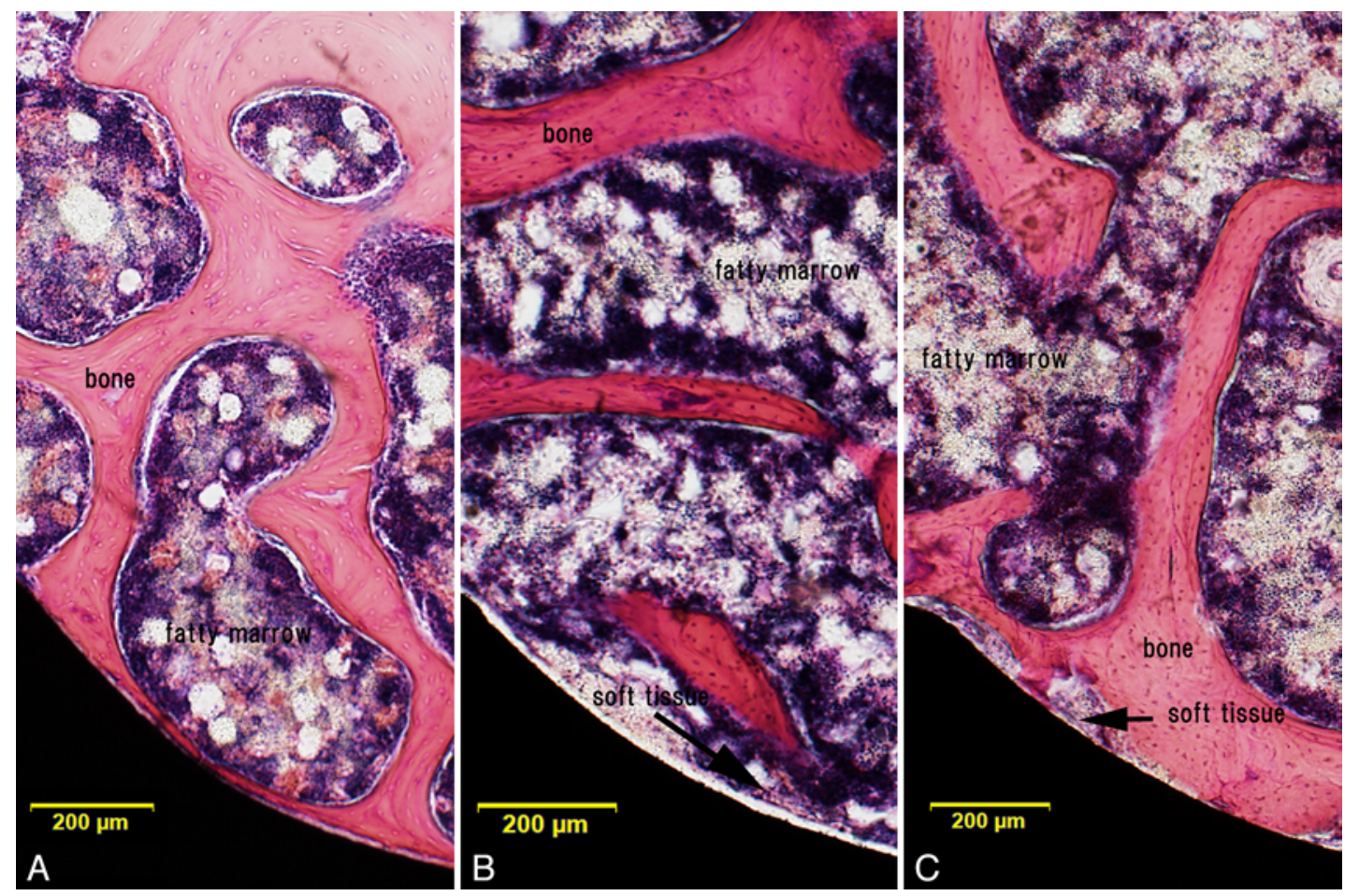

FIG. 6. Qualitative analysis of the BII after 24 weeks. A: HAPSs. The formation of lamellar bone in close contact with the implant is apparent. B: STPSs. Numerous soft-tissue interpositions are present in the gaps between the bone and the implants. C: BLPSs. Some formation of lamellar bone can be seen. At locations farther away from the implants, fatty marrow conversion associated with osteoporosis is present in all of the samples, and no clear difference among the 3 types of screws can be seen. Figure is available in color online only.

the STPSs after 8 and 24 weeks, and good formation of continuous lamellar bone in close contact with the PSs was found, which reflects the previously reported fact that the irregular shape of the IS effectively increases protein adsorption and osteoblast adhesion. ${ }^{6}$ A comparison of the HAPSs and BLPSs revealed that extraction torque values tended to be higher for HAPSs than for BLPSs at 4 weeks and were significantly higher at 8 weeks; however, from 12 weeks onward, the values of HAPSs and BLPSs were similarly high, and the significant difference disappeared. This result indicates that simply coating the surface of the
PS with HA by using the sputtering process, excluding the effect of its surface morphology, increases the rate at which osseointegration is achieved.

Other than after 4 weeks, we found no significant difference in the BV/TV ratios, which indicate the total volume of bone substance surrounding the PSs, between any of the samples in the osteoporosis group. At 4 weeks, it was higher for the HAPSs than for the STPSs because of the effect of lamellar bone formation in close contact with the thread, but at locations farther away from the thread, trabecular thinning similar to that seen around the STPSs

TABLE 4. BS/IS ratios for the 3 types of PSs used

\begin{tabular}{|c|c|c|c|c|c|c|}
\hline \multirow[b]{2}{*}{ Week of Spine Harvest } & \multicolumn{3}{|c|}{ BS/IS Ratio (\%) } & \multicolumn{3}{|c|}{$p$ Value } \\
\hline & HAPSs & BLPSs & STPSs & HAPSs vs BLPSs & HAPSs vs STPSs & BLPSs vs STPSs \\
\hline 2 wks & $14.48 \pm 8.41^{*}$ & $14.62 \pm 13.51$ & $4.06 \pm 2.99$ & 0.953 & $0.003 \dagger$ & 0.123 \\
\hline 4 wks & $58.76 \pm 15.01^{*}$ & $8.82 \pm 5.94$ & $8.39 \pm 8.29$ & 0.539 & $0.001 \dagger$ & 0.999 \\
\hline 8 wks & $63.01 \pm 24.81^{*}$ & $47.56 \pm 25.75^{\star}$ & $17.42 \pm 12.63$ & 0.539 & $0.016 \dagger$ & $0.030 \dagger$ \\
\hline 12 wks & $39.61 \pm 6.99^{*}$ & $29.07 \pm 20.23$ & $19.15 \pm 9.34$ & 0.425 & $0.023 \dagger$ & 0.845 \\
\hline 24 wks & $61.03 \pm 22.69 * \ddagger$ & $35.77 \pm 17.40^{*}$ & $4.04 \pm 7.39$ & $0.025 \dagger$ & $0.0002 \dagger$ & $0.001 \dagger$ \\
\hline 24 wks (control) & $74.06 \pm 12.07^{*} \ddagger$ & $36.60 \pm 15.83^{*}$ & $15.24 \pm 12.81$ & $0.001 \dagger$ & $0.0004 \uparrow$ & $0.014 \dagger$ \\
\hline
\end{tabular}

Ratio values presented as the mean \pm SD.

${ }^{*} p<0.05$ compared with STPSs.

$\dagger$ Significant difference.

$\ddagger p<0.05$ compared with BLPSs. 
TABLE 5. BV/TV ratios for the 3 types of PSs used

\begin{tabular}{lcccc}
\hline \multirow{2}{*}{\begin{tabular}{c} 
Week of $\begin{array}{c}\text { Spine } \\
\text { Harvest }\end{array}$ \\
\cline { 2 - 5 }
\end{tabular}} & HAPSs & BLPSs & \multicolumn{1}{c}{ STPSs } & $\begin{array}{c}\text { PValue } \\
\text { (HAPSs } \\
\text { VS } \\
\text { STPSs) }\end{array}$ \\
\hline 2 wks & $29.40 \pm 3.85$ & $27.46 \pm 6.94$ & $23.37 \pm 7.47$ & 0.153 \\
\hline 4 wks & $53.18 \pm 8.14^{*}$ & $36.36 \pm 8.94$ & $42.61 \pm 8.65$ & 0.042 \\
\hline 8 wks & $46.45 \pm 10.85$ & $54.64 \pm 6.67$ & $51.04 \pm 13.04$ & 0.801 \\
\hline 12 wks & $57.34 \pm 14.81$ & $45.78 \pm 11.77$ & $46.12 \pm 13.43$ & 0.178 \\
\hline 24 wks & $34.46 \pm 15.93$ & $50.31 \pm 14.06$ & $29.83 \pm 9.11$ & 0.729 \\
\hline 24 wks & $52.95 \pm 9.42$ & $68.77 \pm 10.97$ & $62.85 \pm 11.69$ & 0.081 \\
(control) & & & & \\
\hline
\end{tabular}

Ratio values presented as the mean \pm SD.

${ }^{*} p<0.05$ compared with STPSs.

was evident (Fig. 5D and E). This result suggests that the bone conductivity of the thin HA coating could not overcome the effect of osteoporosis on the bone at locations not directly adjacent to the thread.

To prevent loosening of PSs inserted in fragile osteoporotic bone for clinical purposes, not only must the BII be firmly anchored, but the quality of the bone surrounding the PS also must be improved to prevent the occurrence of microfractures. The drugs currently used to treat osteoporosis, bisphosphonates (which inhibit bone resorption) and parathyroid hormone (which promotes bone formation), might help to increase the volume of bone substance around the PSs and prevent fractures ${ }^{16}$ Using the HAPSs in conjunction with osteoporosis drugs might further improve PS fixation in fragile bone, but additional studies are required for verification.

This study had some limitations. First, although we investigated the value of HAPSs at multiple time points in a porcine osteoporosis model, we used only 1 animal to provide a sample at each time point because of the limited scale of our facility and bioethical considerations. In addition to the small sample size, individual differences between the experimental animals might have influenced the outcome. Second, we used only extraction torque tests in the biomechanical testing of PSs. In a 1998 study, Brånemark and $\mathrm{Skalak}^{3}$ stated that extraction torque tests are the best way of evaluating the interface mechanics between an implant and the surrounding tissue, whereas pull-out tests are affected more strongly by the properties of the bone surrounding the implant rather than the interface. Given the limited number of samples, we used only extraction torque tests to concentrate on evaluation of the mechanical stability of the interface. Third, we did not perform $\mathrm{CT}$ of the harvested spines after screw placement. Vertebrae that sustained damage, such as pedicle fractures and cortical breaches of the PS, were excluded without using a microscope. Any damage that we did not detect also might have influenced the outcome of biomechanical testing. Last, we performed stand-alone insertion of the PSs to eliminate the effect of spinal movement on the BII after PS insertion. In clinical spinal instrumentation surgery, the PSs are connected by rods or plates, and spinal fusion and/or deformity-correction surgery is also performed; thus, this experimental model did not fully reproduce a clinical situation. Additional studies under conditions closer to those found in actual clinical practice are required before the HAPSs can be brought into clinical use. The results of our study suggest that stand-alone placement of PSs before definitive stabilization surgery might be a possible alternative method for performing spinal reconstructive surgery for patients with an osteoporotic spine.

\section{Conclusions}

In our in vivo experimental study using a porcine model of postmenopausal osteoporosis, PSs with a thin HA coating applied by using the sputtering process provided stronger fixation at the BII where the screw and bone substance are in close contact, which suggests that they might be able to reduce the incidence of complications caused by loosening of PSs after spinal instrumentation surgery. However, the absence of increased bone mass around the screw remains an issue; improving bone quality by prescribing osteoporosis treatment might be necessary to prevent fractures around the screws.

\section{References}

1. Aldini NN, Fini M, Giavaresi G, Giardino R, Greggi T, Parisini P: Pedicular fixation in the osteoporotic spine: a pilot in vivo study on long-term ovariectomized sheep. J Orthop Res 20:1217-1224, 2002

2. Brånemark R, Ohrnell LO, Skalak R, Carlsson L, Brånemark PI: Biomechanical characterization of osseointegration: an experimental in vivo investigation in the beagle dog. J Orthop Res 16:61-69, 1998

3. Brånemark R, Skalak R: An in-vivo method for biomechanical characterization of bone-anchored implants. Med Eng Phys 20:216-219, 1998

4. Chappard D, Aguado E, Huré G, Grizon F, Basle MF: The early remodeling phases around titanium implants: a histomorphometric assessment of bone quality in a 3-and 6-month study in sheep. Int J Oral Maxillofac Implants 14:189-196, 1999

5. Chin DK, Park JY, Yoon YS, Kuh SU, Jin BH, Kim KS, et al: Prevalence of osteoporosis in patients requiring spine surgery: incidence and significance of osteoporosis in spine disease. Osteoporos Int 18:1219-1224, 2007

6. Correa MG, Gomes Campos ML, César-Neto JB, Casati MZ, Nociti FH, Sallum EA: Histometric evaluation of bone around titanium implants with different surface treatments in rats exposed to cigarette smoke inhalation. Clin Oral Implants Res 20:588-593, 2009

7. Feighan JE, Goldberg VM, Davy D, Parr JA, Stevenson S: The influence of surface-blasting on the incorporation of titanium-alloy implants in a rabbit intramedullary model. J Bone Joint Surg Am 77:1380-1395, 1995

8. Ferguson RL, Tencer AF, Woodard P, Allen BL Jr: Biomechanical comparisons of spinal fracture models and the stabilizing effects of posterior instrumentations. Spine (Phila Pa 1976) 13:453-460, 1988

9. Halvorson TL, Kelley LA, Thomas KA, Whitecloud TS III, Cook SD: Effects of bone mineral density on pedicle screw fixation. Spine (Phila Pa 1976) 19:2415-2420, 1994

10. Galbusera F, Volkheimer D, Reitmaier S, Berger-Roscher N, Kienle A, Wilke HJ: Pedicle screw loosening: a clinically relevant complication? Eur Spine J 24:1005-1016, 2015

11. Hasegawa T, Inufusa A, Imai Y, Mikawa Y, Lim TH, An HS: Hydroxyapatite-coating of pedicle screws improves resis- 
tance against pull-out force in the osteoporotic canine lumbar spine model: a pilot study. Spine J 5:239-243, 2005

12. Hitchon PW, Brenton MD, Black AG, From A, Harrod JS, Barry C, et al: In vitro biomechanical comparison of pedicle screws, sublaminar hooks, and sublaminar cables. J Neurosurg 99 (1 Suppl): 104-109, 2003

13. Jansen JA, van der Waerden JP, Wolke JG: Histologic investigation of the biologic behavior of different hydroxyapatite plasma-sprayed coatings in rabbits. J Biomed Mater Res 27:603-610, 1993

14. Lai KA, Shen WJ, Chen CH, Yang CY, Hu WP, Chang GL: Failure of hydroxyapatite-coated acetabular cups. Ten-year follow-up of 85 Landos Atoll arthroplasties. J Bone Joint Surg Br 84:641-646, 2002

15. Mosekilde L, Weisbrode SE, Safron JA, Stills HF, Jankowsky ML, Ebert DC, et al: Calcium-restricted ovariectomized Sinclair S-1 minipigs: an animal model of osteopenia and trabecular plate perforation. Bone 14:379-382, 1993

16. Ohtori S, Inoue G, Orita S, Yamauchi K, Eguchi Y, Ochiai N, et al: Comparison of teriparatide and bisphosphonate treatment to reduce pedicle screw loosening after lumbar spinal fusion surgery in postmenopausal women with osteoporosis from a bone quality perspective. Spine (Phila Pa 1976) 38:E487-E492, 2013

17. Onsten I, Nordqvist A, Carlsson AS, Besjakov J, Shott S: Hydroxyapatite augmentation of the porous coating improves fixation of tibial components. A randomised RSA study in 116 patients. J Bone Joint Surg Br 80:417-425, 1998

18. Ozeki K, Mishima A, Yuhta T, Fukui Y, Aoki H: Bone bonding strength of sputtered hydroxyapatite films subjected to a low temperature hydrothermal treatment. Biomed Mater Eng 13:451-463, 2003

19. Ozeki K, Yuhta T, Aoki H, Nishimura I, Fukui Y: Crystal chemistry of hydroxyapatite deposited on titanium by sputtering technique. Biomed Mater Eng 10:221-227, 2000

20. Piattelli A, Scarano A, Di Alberti L, Piattelli M: Bonehydroxyapatite interface in retrieved hydroxyapatite-coated titanium implants: a clinical and histologic report. Int J Oral Maxillofac Implants 14:233-238, 1999

21. Shiba K, Taneichi H, Namikawa T, Inami S, Takeuchi D, Nohara Y: Osseointegration improves bone-implant interface of pedicle screws in the growing spine: a biomechanical and histological study using an in vivo immature porcine model. Eur Spine J 26:2754-2762, 2017

22. Wu ZX, Gong FT, Liu L, Ma ZS, Zhang Y, Zhao X, et al: A comparative study on screw loosening in osteoporotic lumbar spine fusion between expandable and conventional pedicle screws. Arch Orthop Trauma Surg 132:471-476, 2012

23. Xie Y, Fu Q, Chen ZQ, Shi ZC, Zhu XD, Wang CF, et al: Comparison between two pedicle screw augmentation instrumentations in adult degenerative scoliosis with osteoporosis. BMC Musculoskelet Disord 12:286, 2011

\section{Disclosures}

H.T. has received speaker's bureau fees from Century Medical, Inc. No funding was provided for any portion of this research product.

\section{Author Contributions}

Conception and design: Nohara, Taneichi. Acquisition of data: Moridaira, Ohe. Analysis and interpretation of data: Moridaira, Ohe, Taneichi. Drafting the article: Moridaira. Critically revising the article: Moridaira, Taneichi. Reviewed submitted version of manuscript: all authors. Approved the final version of the manuscript on behalf of all authors: Moridaira. Statistical analysis: Moridaira, Ohe. Administrative/technical/material support: all authors. Study supervision: Nohara, Taneichi.

\section{Correspondence}

Hiroshi Moridaira: Dokkyo Medical University, Tochigi, Japan. morihey@dokkyomed.ac.jp. 